\title{
High UV Sensitivity of SiON Film and Its Application to Center Wavelength Trimming of Microring Resonator Filter
}

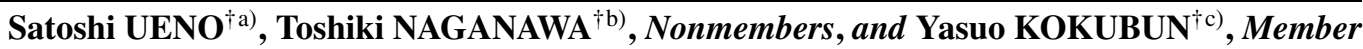

\begin{abstract}
SUMMARY We demonstrated a vertically coupled microring resonator (VCMRR) filter as an Add/Drop wavelength filter. However, the accuracy of center wavelength was not sufficiently high for dense wavelength division multiplexing (DWDM) systems. Thus, a UV trimming technique using a $\mathrm{SiN}(n=2.01$ at $\lambda=1.55 \mu \mathrm{m})$ ring core was previously developed. Although a wide center wavelength trimming range of $-12.1 \mathrm{~nm}$ and the long-term stability of center wavelength were realized, the core size required for single-mode propagation was too small for fabrication using a photolithography process. Therefore in this study, we introduced SiON as the microring core to relax the single-mode condition of core size. We discovered a large UV sensitivity of SiON film formed by a PECVD method, and a wide range UV trimming of microring resonator of $-10.5 \mathrm{~nm}$ was demonstrated using this phenomenon.

key words: microring resonator, UV sensitivity, trimming, SiON
\end{abstract}

\section{Introduction}

A vertically coupled microring resonator (VCMRR) is an attractive Add/Drop wavelength filter for a WDM access network [1]-[3] due to its functionality, compactness, and the possibility of dense integration owing to a cross-grid configuration. In this stacked configuration, a microring resonator is placed at the crossing point of busline waveguide and add-drop waveguides, which are crossed at right angles, as shown in Fig. 1. The upper layer acts as the wavelengthselective waveguide layer with a high index contrast (typically $35-37 \%$ ), which yields a very small ring radius (typically $10-20 \mu \mathrm{m}$ ) with a high Q factor, while the lower layer acts as the busline waveguide layer, which is designed for better coupling to single-mode fibers. Due to the small ring radius, the FSR is expanded to several tens of $\mathrm{nm}$, which covers the bandwidth of EDFA. Due to the cross-grid topology and the small ring radius, a dense integration like $10^{4}$ to $10^{5}$ devices $/ \mathrm{cm}^{2}$ will be possible. In addition, in the vertical coupling configuration, the coupling coefficient is precisely determined by the thickness control of buffer layer, which is much easier than the gap control by etching in the case of parallel coupling. However, the error of resonant wavelength from the designed value occurs due to the nonuniformity of index and thickness of film and fabrication error.

In solving this problem, we discovered that the UV sensitivity of SiN film formed by the plasma-enhanced chemi-

Manuscript received September 29, 2004.

Manuscript revised January 19, 2005.

${ }^{\dagger}$ The authors are with the Graduate School of Eng., Yokohama National Univ., Yokohama-shi, 240-8501 Japan.

a)E-mail: ueno@koklab.seg.ynu.ac.jp

b)E-mail: naganawa@koklab.seg.ynu.ac.jp

c)E-mail: kokubun@ynu.ac.jp

DOI: $10.1093 /$ ietele/e88-c.5.998

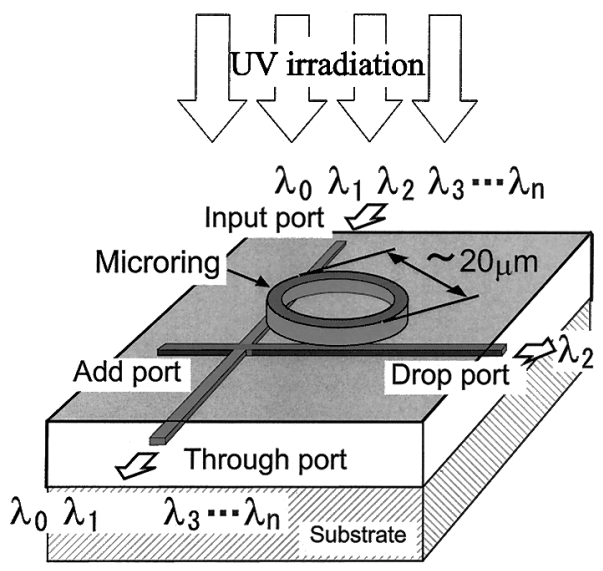

Fig. 1 Principle of center wavelength trimming by direct UV irradiation to ring core.

cal vapor deposition (PECVD) method was much larger than that of Ge-doped $\mathrm{SiO}_{2}$ and demonstrated a wide range UV trimming of center wavelength of $-12.1 \mathrm{~nm}$ using this phenomenon [4]. Since the refractive index of $\mathrm{SiN}$ was 2.0, the relative index difference was as large as $38 \%$ using an air cladding layer. This large index contrast was considered suitable for realizing a small ring radius of less than $10 \mu \mathrm{m}$. However, the core size required for single-mode propagation was too small for fabrication using a photolithography process.

Therefore in this study, we introduced SiON $(n=1.74$ 1.82 ) as the microring core to relax the single-mode condition of core size, and measured the UV sensitivity for the trimming of VCMRR, as shown in Fig. 1.

\section{Principle of Center Wavelength Trimming}

The resonant wavelength of microring resonator filter is mainly determined by the ring radius and the equivalent index. The shift of center wavelength due to the change in equivalent index is determined by

$$
\Delta \lambda_{c}=\frac{2 \pi R \Delta n_{e q}}{N},
$$

where $R$ is the ring radius, $N$ is the resonance order and $\mathrm{n}_{e q}$ is the equivalent index. When the refractive index of ring waveguide is changed by UV irradiation, the equivalent index is also changed. Thus, the center wavelength is shifted. 


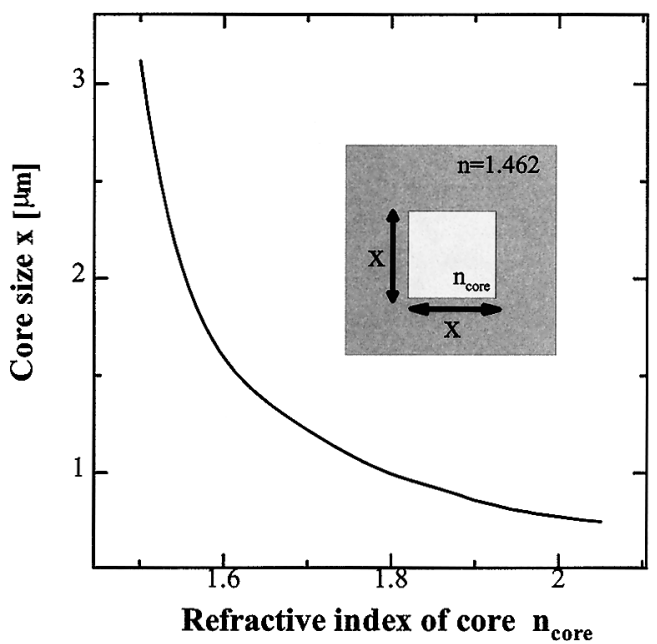

Fig. 2 Single-mode condition of square core waveguide $(\lambda=1550 \mathrm{~nm})$.

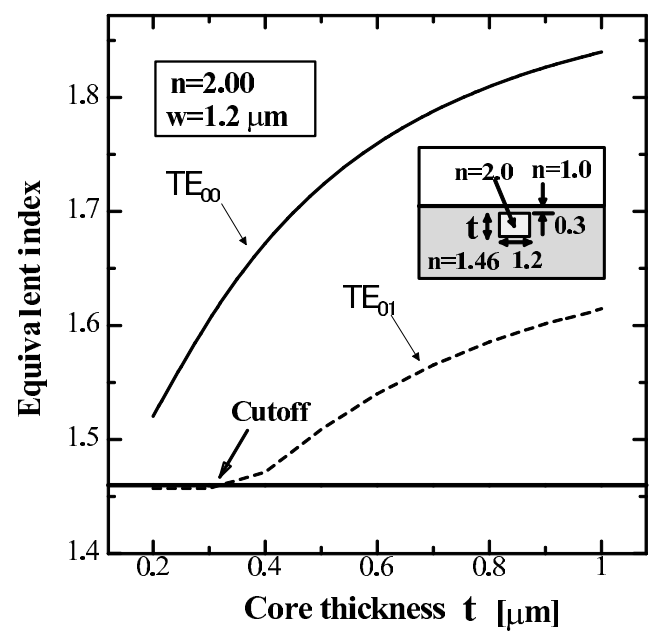

Fig. 3 Single-mode condition of busline waveguide with $\mathrm{SiN}$ core $(\lambda=$ $1550 \mathrm{~nm})$.

\section{Analysis of Single-Mode Condition}

To relax the core size required for single-mode propagation, the refractive index in the core was reduced. First, we analyzed the single-mode condition for the square core waveguide. The result is shown in Fig. 2. In this analysis, the finite-element mode solver supplied by Appolo Optics (APSS) was used. The transverse electric (TE) mode was assumed, because the single-mode condition for the TE mode is smaller than that for the transverse magnetic (TM) mode.

When the refractive index is 2.0 , the core size required for single-mode propagation is $0.8 \mu \mathrm{m}$ width $\times 0.8 \mu \mathrm{m}$ height. On the other hand, when we decrease the refractive index down to 1.7 , the single-mode condition of core size is relaxed to $1.21 \mu \mathrm{m}$ width $\times 1.21 \mu \mathrm{m}$ height, which is a relaxation of $50 \%$. Therefore, the core size required for the single-mode conditon can be relaxed by reducing the refractive index.

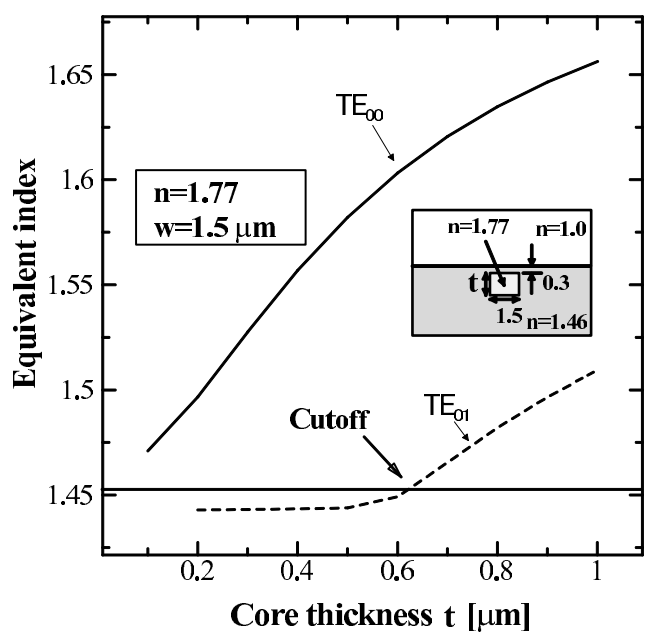

Fig. 4 Single-mode condition of busline waveguide with SiON core $(\lambda=$ $1550 \mathrm{~nm})$.

Next, to design the actual waveguide structure, we analyzed the single-mode condition of busline waveguide of which core is made of $\mathrm{SiN}$ with a refractive index of 2.0 and SiON with a refractive index of 1.77 . The singlemode condition of straight busline waveguide is more severe than that of ring waveguide with a small ring radius. The single-mode condition of busline waveguide made of SiN is shown in Fig. 3 and that of SiON busline core is shown in Fig. 4. When SiN is used as the core material, the core size required for the single-mode condition is $1.2 \mu \mathrm{m}$ width $\times 0.3 \mu \mathrm{m}$ height, which is too small for photolithography using a contact-type mask aligner in our experimental facility. On the other hand, when we decrease the refractive index down to 1.77 , the single-mode condition of core size is relaxed to $1.5 \mu \mathrm{m}$ width $\times 0.6 \mu \mathrm{m}$ height, as shown in Fig. 4 . Thus, we introduced the SiON film as the core material in our experiment.

\section{Refractive Index Control of SiON Film by PECVD}

We deposited a SiON film on a Si substrate by a prasmaenhanced chemical vapor deposition method. The source gas of $\mathrm{Si}$ was $\mathrm{SiH}_{4}$ or $\mathrm{Si}\left(\mathrm{CH}_{3}\right)_{4}$ and reactant gases were $\mathrm{NH}_{3}, \mathrm{~N}_{2}, \mathrm{Ar}$, and $\mathrm{N}_{2} \mathrm{O}$. The total pressure was $1.2 \mathrm{~Pa}$, the RF $(13.56 \mathrm{MHz})$ power was $250 \mathrm{~W}$, the $\mathrm{LF}(400 \mathrm{kHz})$ power supplied to the substrate was $100 \mathrm{~W}$, and the substrate temperature was $300^{\circ} \mathrm{C}$. Three different films, i.e., $\mathrm{SiN}, \mathrm{SiON}$ and $\mathrm{SiO}_{2}$, were deposited using this apparatus. For the deposition of $\mathrm{SiN}$ film, $\mathrm{N}_{2} \mathrm{O}$ was eliminated from the above combination of reactant gases, and for the deposition of $\mathrm{SiO}_{2}$ film, $\mathrm{NH}_{3}$ and $\mathrm{N}_{2}$ were eliminated. When the $\mathrm{SiON}$ film was deposited, all the above-mentioned gases were used. As for the source gas of $\mathrm{Si}$, we used two different materials, i.e., $\mathrm{SiH}_{4}$ and $\mathrm{Si}\left(\mathrm{CH}_{3}\right)_{4}$, and the differences in optical characteristics between the films formed using these source gases are discussed in Sect. 5.2.

The controllability of the refractive index of SiON film was examined by changing the flow rate of $\mathrm{N}_{2} \mathrm{O}$ gas. In 
Table 1 Deposition conditions.

\begin{tabular}{|c|c|c|c|c|c|}
\hline Gas & $\mathrm{SiH}_{4}$ & $\mathrm{~N}_{2}$ & $\mathrm{NH}_{3}$ & $\mathrm{Ar}$ & $\mathrm{N}_{2} \mathrm{O}$ \\
\hline Unit & $\mathrm{sccm}$ & $\mathrm{sccm}$ & $\mathrm{sccm}$ & $\mathrm{sccm}$ & $\mathrm{sccm}$ \\
\hline Flow rate & 50 & 100 & 100 & 200 & $x$ \\
\hline
\end{tabular}

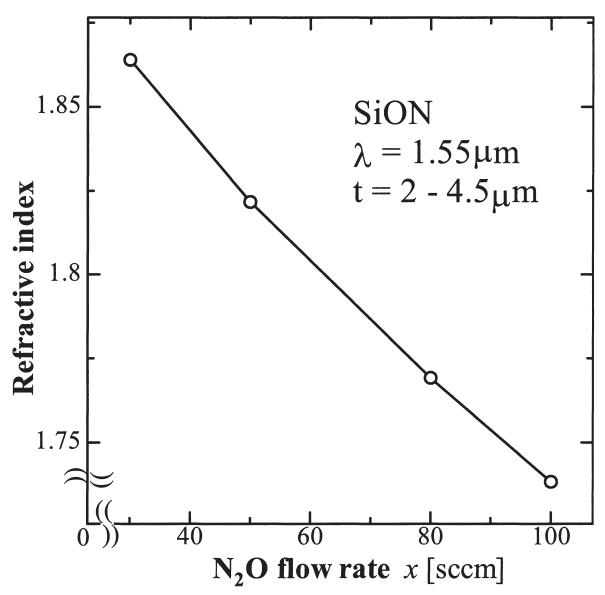

Fig. 5 Refractive index of $\mathrm{SiON}$ vs. $\mathrm{N}_{2} \mathrm{O}$ flow rate.

this experiment, only the flow rate of $\mathrm{N}_{2} \mathrm{O}$ was changed and those of other gases were kept constant, as shown in Table 1. The measured result is shown in Fig. 5. The refractive index was measured using a prism coupler (Metricon PC-2010). It can be seen that the refractive index decreases almost linearly with the increase in the flow rate of $\mathrm{N}_{2} \mathrm{O}$. Thus, the refractive index was controlled by the $\mathrm{N}_{2} \mathrm{O}$ flow rate in the range of 1.74-1.86.

\section{Refractive Index Change of SiON Film by UV Irra- diation}

We measured the UV-induced refractive index change of $\mathrm{SiON}$ film, which was deposited on a Si substrate by the plasma-enhanced CVD method using $\mathrm{SiH}_{4}$. A Hg lamp was used as the UV source, and the wavelength and the power density were $254 \mathrm{~nm}$ and $42.6 \mathrm{~mW} / \mathrm{cm}^{2}$, respectively.

\subsection{Comparison of UV Sensitivity of SiN and SiON Films}

Figure 6 shows a comparison of refractive index changes of $\mathrm{SiN}$ and $\mathrm{SiON}$ films against UV irradiation time. For 9-h irradiation, the index of $\mathrm{SiN}$ of 2.03 decreased by $-2.2 \times$ $10^{-3}$. In contrast, the index of $\mathrm{SiON}$ of 1.77 decreased by $-6.4 \times 10^{-3}$. It was observed that the refractive index change of $\mathrm{SiON}$ is much faster and greater than that of $\mathrm{SiN}$.

\subsection{Comparison of UV Sensitivities of SiON Films Formed Using Different Source Gases}

$\mathrm{SiN}$ and SiON films were formed using two different source gases, $\mathrm{SiH}_{4}$ and $\mathrm{Si}\left(\mathrm{CH}_{3}\right)_{4}$, in our PECVD apparatus. The controllable range of refractive index was almost the same for these two materials. Thus, the UV sensitivities of SiON films deposited using these materials were measured and

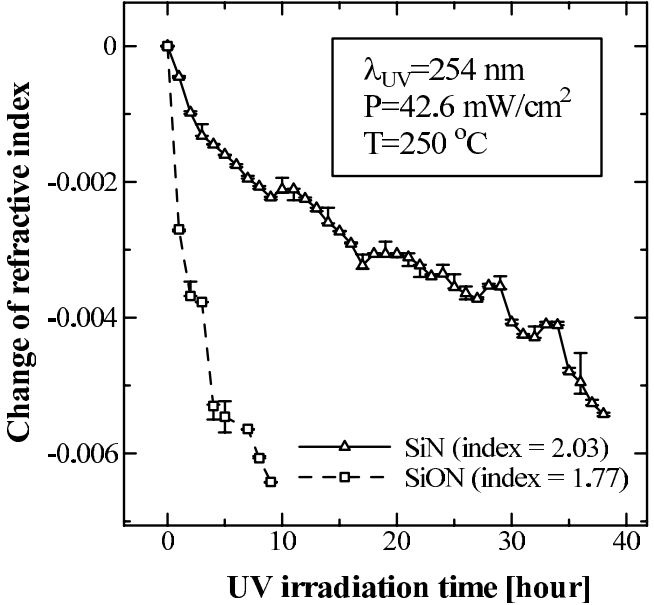

Fig. 6 Comparison of UV sensitivities of $\mathrm{SiN}$ and $\mathrm{SiON}$ films $(\lambda=$ $1550 \mathrm{~nm})$.

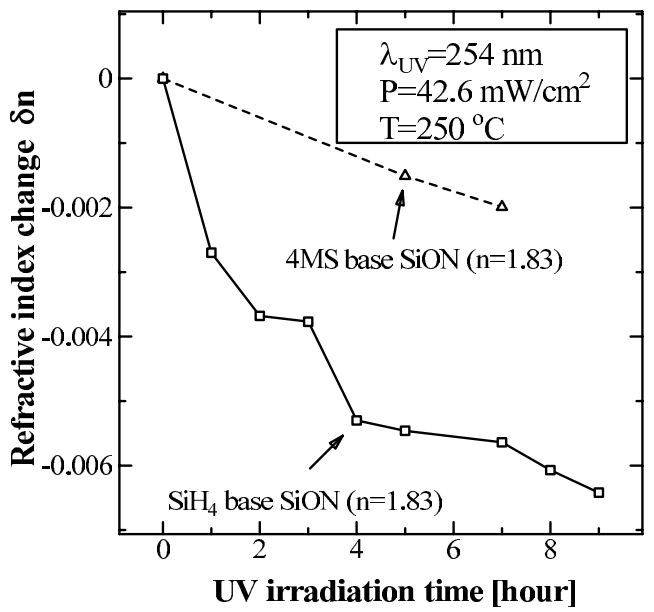

Fig. 7 Comparison of UV sensitivities of $\mathrm{SiON}$ films made from $\mathrm{SiH}_{4}$ and $\mathrm{Si}\left(\mathrm{CH}_{3}\right)_{4}(\lambda=1550 \mathrm{~nm})$.

compared. The results are shown in Fig. 7. For 7-h irradiation, the index of $\mathrm{SiON}$ made from $\mathrm{Si}\left(\mathrm{CH}_{3}\right)_{4}$ (tetramethylsilane) decreased by $-1.9 \times 10^{-3}$. In contrast, the index of $\mathrm{SiON}$ made from $\mathrm{SiH}_{4}$ (monosilane) decreased by $-5.6 \times 10^{-3}$. The refractive index change of $\mathrm{SiON}$ made from $\mathrm{SiH}_{4}$ was greater than that made from $\mathrm{Si}\left(\mathrm{CH}_{3}\right)_{4}$. Therefore, $\mathrm{SiON}$ made from $\mathrm{SiH}_{4}$ was used as the ring core material.

\subsection{Comparison of UV Sensitivities of SiON Films against Initial Refractive Index}

The refractive index change of SiON films of different initial refractive indices $(n=1.74,1.77,1.82$ and 1.86$)$ was measured to examine the relationship between the refractive index change after UV irradiation and that before UV irradiation. In the preparation of SiON films, only the flow rate of $\mathrm{N}_{2} \mathrm{O}$ was changed to control the refractive index and the conditions of the other gases were maintained constant so that the film quality would not significantly change. The result is shown in Fig. 8. The refractive index of four $\mathrm{SiON}$ 
films changed at an almost equal rate against the UV irradiation time for the first $5 \mathrm{~h}$. After 5-h irradiation, the index change of SiON film with a lower initial refractive index saturated faster and the maximum index change of 0.036 was obtained for the SiON film with the initial refractive index of 1.86 for the irradiation time of $80 \mathrm{~h}$. This index change of 0.036 is about fourfold greater than that of Ge-doped silica glass.

\subsection{Stability after UV Irradiation}

We measured the stability of refractive index change after UV irradiation. The result is shown in Fig. 9. The refractive index was almost stable for more than $250 \mathrm{~h}$ after UV irradiation. This result is similar to that obtained for the SiN film [4]. Thus, it was expected that the center wavelength after UV trimming would also be stable without any treatment such as hydrogen loading.

The origin of the UV-induced refractive index change was not completely analyzed. However, since the SiON film

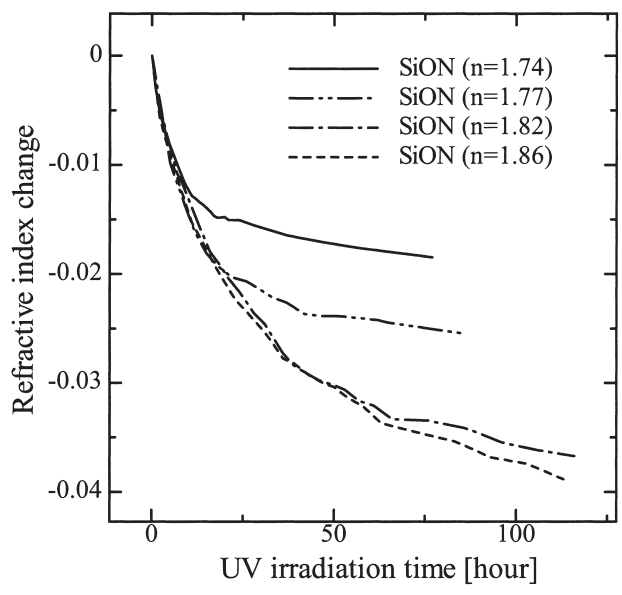

Fig. 8 Comparison of UV sensitivities of SiON films against initial refractive index $(\lambda=1550 \mathrm{~nm})$.

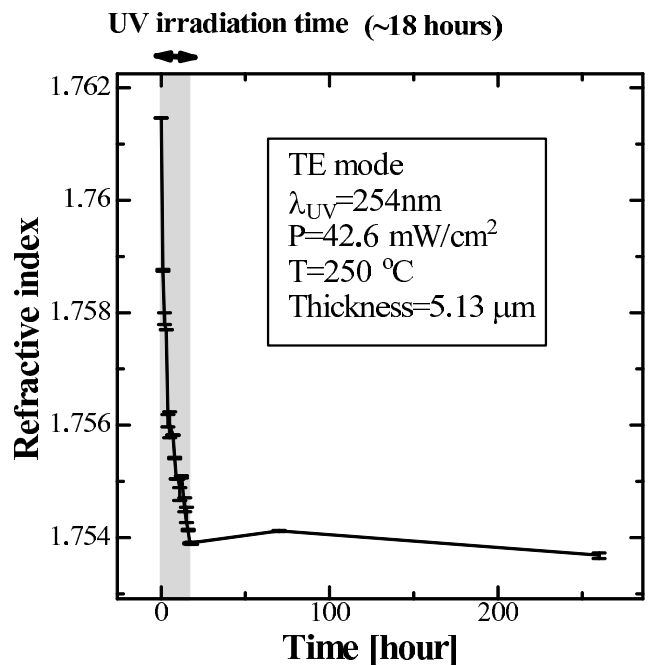

Fig. 9 Stability of index change after UV irradiation. was deposited using monosilane and ammonia gases and the film was expected to contain certain amounts of hydrogen and nytrogen, the UV-induced index change was considered to be caused by the change in $\mathrm{Si}-\mathrm{H}$ and $\mathrm{N}-\mathrm{H}$ bonds due to UV exposure.

\section{Fabrication of Vertically Coupled Microring Res- onator Filter with SiON Ring Core}

\subsection{Fabrication Process}

Figure 10 shows the fabrication process of vertically coupled microring resonator. Although the single-mode condition was analyzed using the rectangular waveguide, we adopted the ridge waveguide as the busline waveguide. This is because the single-mode condition of ridge waveguide is larger than that of rectangular waveguide with the same core thickness and core width. In addition, the realization of flat top surface is easier using the ridge structure than using the rectangular waveguide, because the ridge with a height of $0.2 \mu \mathrm{m}$ can be buried by the spin coating of spin-on-glass (SOG).

A $\mathrm{SiO}_{2}$ film used as the cladding layer was deposited on a $\mathrm{Si}$ substrate by the thermal oxidation method, and a $\mathrm{SiON}$ film used as the core of busline waveguide was deposited by the PECVD method. The thicknesses of $\mathrm{SiO}_{2}$ cladding and SiON busline core were $7.5 \mu \mathrm{m}$ and $0.5 \mu \mathrm{m}$,

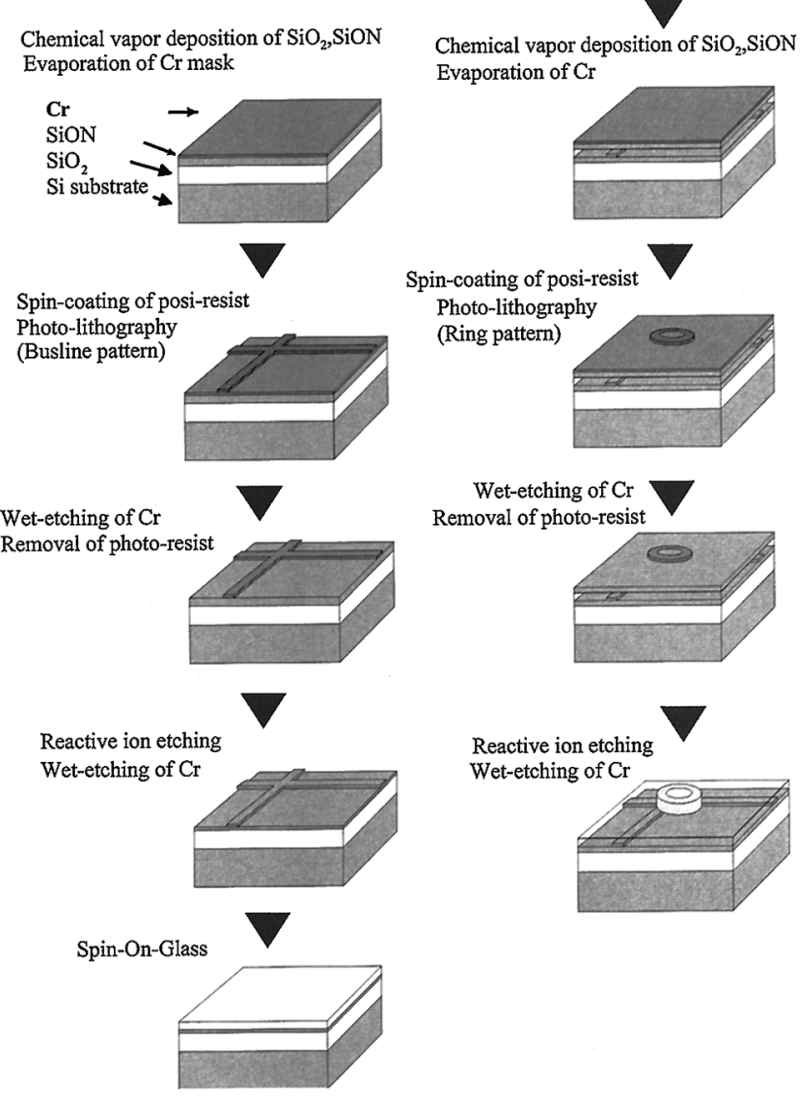

Fig. 10 Fabrication process. 


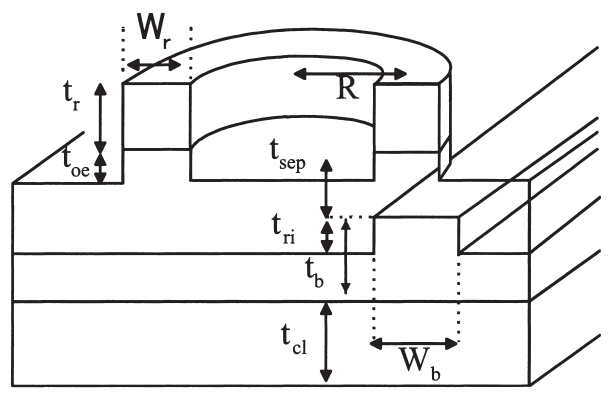

Fig. 11 Structure of vertically coupled microring resonator filter.

Table 2 Dimensions of device.

\begin{tabular}{|c|c|c|}
\hline & symbol & dimensions \\
\hline Ring radius & $\mathrm{R}$ & $20 \mu \mathrm{m}$ \\
\hline Ring waveguide width & $\mathrm{W}_{r}$ & $1.5 \mu \mathrm{m}$ \\
\hline Busline waveguide width & $\mathrm{W}_{b}$ & $1.5 \mu \mathrm{m}$ \\
\hline Ring waveguide thickness & $\mathrm{t}_{r}$ & $0.9 \mu \mathrm{m}$ \\
\hline Busline waveguide ridge height & $\mathrm{t}_{r i}$ & $0.3 \mu \mathrm{m}$ \\
\hline Thickness of busline core & $\mathrm{t}_{b}$ & $0.5 \mu \mathrm{m}$ \\
\hline Separation layer thickness & $\mathrm{t}_{s e p}$ & $0.5 \mu \mathrm{m}$ \\
\hline Over etching depth & $\mathrm{t}_{o e}$ & $0.2 \mu \mathrm{m}$ \\
\hline Lower cladding layer & $\mathrm{t}_{c l}$ & $7.5 \mu \mathrm{m}$ \\
\hline
\end{tabular}

respectively. Next, 100-nm-thick $\mathrm{Cr}$ was deposited as the mask for reactive ion etching (RIE). Then, the busline waveguide was formed using the photolithography process and RIE with $\mathrm{C}_{2} \mathrm{~F}_{6}$ gas, and the $\mathrm{Cr}$ mask was removed by the wet chemical etching. Next, the step height was eliminated by the spin coating of spin-on-glass (SOG). After the baking of SOG, the top surface of the busline waveguide was perfectly planarized. Since the typical thickness of SOG layer was $0.2 \mu \mathrm{m}$ and the required thickness of buffer layer between the busline core and the ring core ranged from 0.5 to $0.7 \mu \mathrm{m}$, an additional $\mathrm{SiO}_{2}$ buffer layer of $0.3-0.5 \mu \mathrm{m}$ thickness was deposited. Then the $\mathrm{SiON}$ ring core layer was deposited by the PECVD method. Next, Cr was deposited, and the ring waveguide was formed using the photolithography process and RIE.

We fabricated eight ring resonators of identical ring radius within the area of $2 \mathrm{~mm} \times 300 \mu \mathrm{m}$ to measure the distribution of center wavelength resulting from the fabrication error. The cross-sectional structure denoted by symbols is shown in Fig. 11, and the dimensions of each part are shown in Table 2.

\subsection{Filter Characteristics}

Figure 12 shows the measured drop and through port characteristics of one of the eight ring resonators with the ring radius of $20 \mu \mathrm{m}$. FSR was $10.32 \mathrm{~nm}$ and FWHM was $1.42 \mathrm{~nm}$. It was observed that single-mode propagation is realized as expected from the designed structure. The small peaks around the wavelengths of $1560 \mathrm{~nm}$ and $1570 \mathrm{~nm}$ seem to be caused by polarization mixing.

In addition, it was observed that the output power level in the wavelength range from $1500 \mathrm{~nm}$ to $1540 \mathrm{~nm}$ is lower

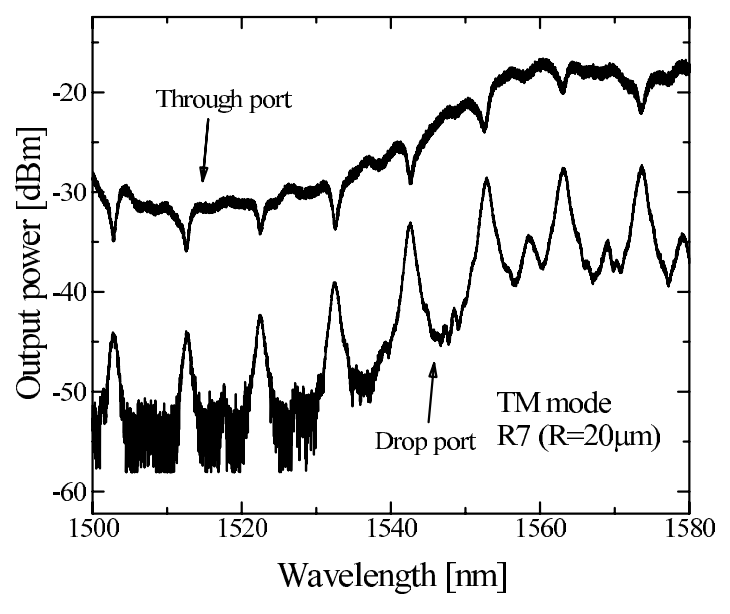

Fig. 12 Filter response.

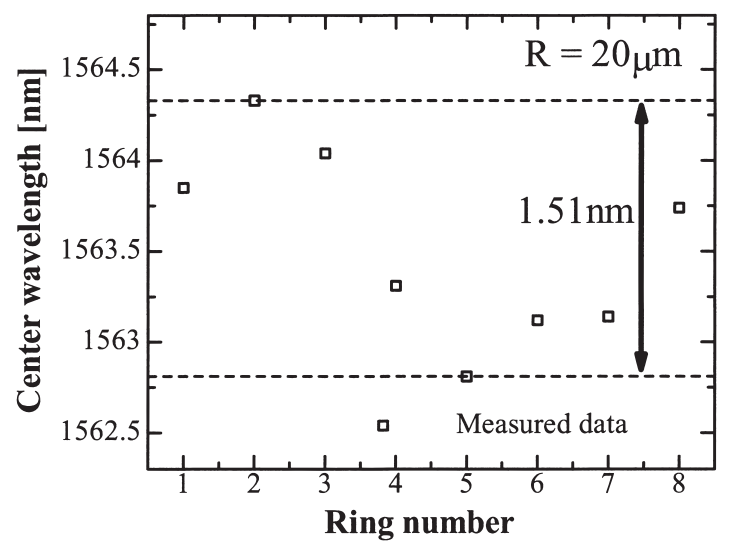

Fig. 13 Fabrication accuracy of VCMRR filter.

than that in the longer wavelength range. This seems to be caused by the absorption loss due to the $\mathrm{N}-\mathrm{H}$ radical, and this absoption is expected to be improved by annealing up to $1160^{\circ} \mathrm{C}$ [5], [6].

\subsection{Fabrication Accuracy of Vertically Coupled Microring Resonator Filter}

The accuracy of center wavelength was measured using the eight microring resonators of identical ring radius fabricated on the same wafer. The result is shown in Fig. 13. The maximum center wavelength difference was $1.51 \mathrm{~nm}$. This error seems to be caused by the fabrication error, particularly the error of the waveguide width due to the accuracy limit of the photolithography process. It was observed from this measurement that a trimming range greater than $2 \mathrm{~nm}$ is required to compensate for the fabrication error.

\section{Center Wavelength Trimming by UV Irradiation}

We fabricated a number of microring resonators using $\mathrm{SiON}$ as the core material using the process shown in Fig. 10, and measured the change in filter response by UV irradiation. 


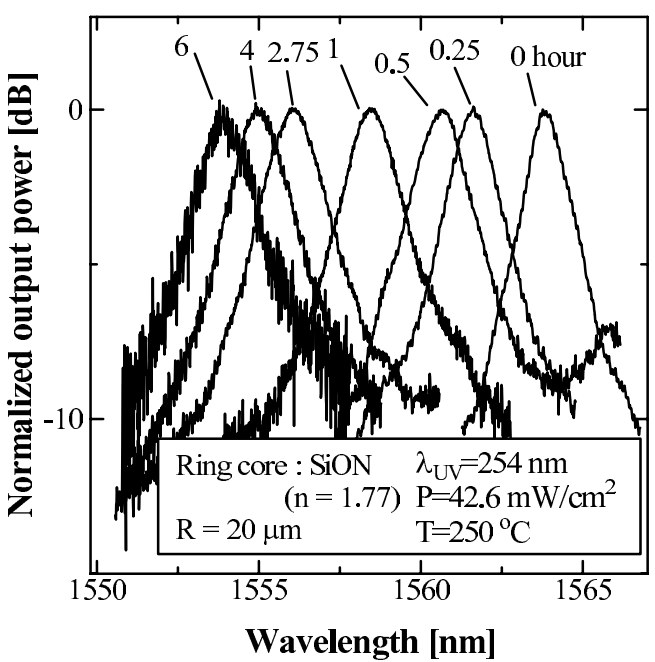

Fig. 14 Change in filter response of VCMRR with SiON core vs. UV irradiation time.

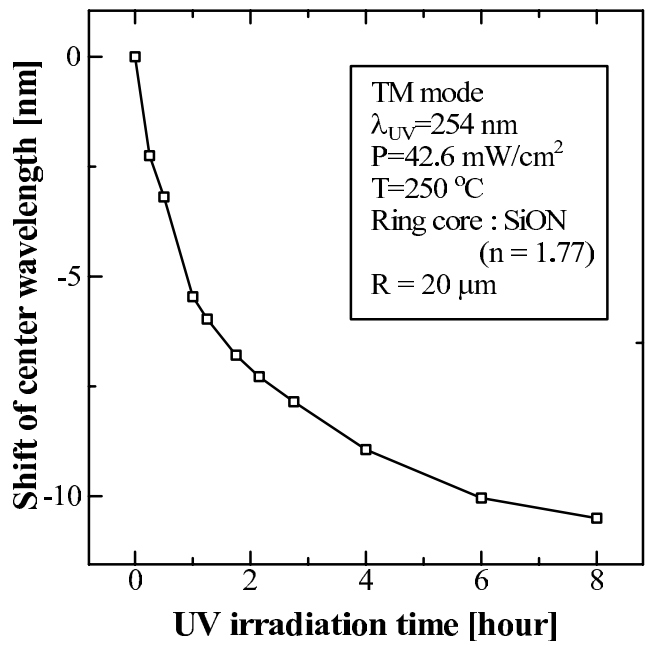

Fig. 15 Shift of center wavelength of VCMRR with SiON core vs. UV irradiation time.

Figure 14 shows the change in the filter response of the vertically coupled microring resonator with a $\mathrm{SiON}$ core against UV irradiation time. The center wavelength shift against the UV irradiation time is shown in Fig. 15. For 8-h UV irradiation, the maximum center wavelength shift was $-10.5 \mathrm{~nm}$. Thus a wide trimming range was successfully demonstrated.

The FWHM bandwidth was increased by $0.38 \mathrm{~nm}$ from $1.52 \mathrm{~nm}$ to $1.9 \mathrm{~nm}$, as shown in Fig. 14. This implied that the propagation loss in the ring increases. However, the exact number of propagation loss could not be obtained, because a clear through port response was not obtained.

In our experiment, a clear TE mode response was not obtained, and thus, the polarization dependence of UV sensitivity was not observed. According to our previous experiment, the polarization dependence of UV sensitivity was about $4 \%$ [4].

\section{Conclusion}

In this study, we aimed at developing a vertically coupled microring resonator using a high index contrast waveguide with a relaxed single-mode condition suitable for photolithography, and also a wide-range UV trimming technique. To achieve this objective, we adopted SiON deposited by a plasma-enhanced CVD method as the core material of busline and microring waveguides, and measured the UV sensitivity. First, the controllability of refractive index was investigated and it was clarified that the refractive index of $\mathrm{SiON}$ film is controlled by changing only the gas flow rate of $\mathrm{N}_{2} \mathrm{O}$. The numerical calculation showed that the use of SiON film relaxes the single-mode condition by $20 \%$ compared with SiN.

Next, the UV sensitivity of SiON film was measured using SiON films of different initial refractive indices, and the maximum index change of 0.036 was obtained for the $\mathrm{SiON}$ film with the refractive index of 1.86 . The stability of index change after UV irradiation was also measured and the index change was confirmed to be stable for more than $250 \mathrm{~h}$ without any treatment.

Using this large UV sensitivity of SiON film, the UV trimming of center wavelength of vertically coupled microring resonator with a $\mathrm{SiON}$ ring core was demonstrated, and a wide trimming range of $-10.5 \mathrm{~nm}$ was successfully obtained.

\section{Acknowledgments}

This work was supported in part by the Grant-in-Aid for Creative Research No.14GS0212 from the Ministry of Education, Culture, Sports, Science and Technology, Futaba Electronics Memorial Foundation, and the 21st Century COE Program of the Ministry of Education, Culture, Sports, Science and Technology.

\section{References}

[1] B.E. Little, S.T. Chu, W. Pan, D. Ripin, T. Kaneko, Y. Kokubun, and E. Ippen, "Vertically coupled glass microring resonator channel dropping filters," Photonics Technol. Lett., vol.11, no.2, pp.215-217, 1999.

[2] S.T. Chu, B. Little, W. Pan, T. Kaneko, S. Sato, and Y. Kokubun, "An eight channel Add/Drop alter using vertically coupled microring resonators over a cross grid," Photonics Technol. Lett., vol.11, no.6, pp.691-693, 1999.

[3] D.V. Tishinin, P.D. Dapkus, A.E. Bond, I. Kim, C.K. Lin, and J. O'Brien, "Vertical resonant couplers with precise coupling efficiency control fabricated by wafer bonding," Photonics Technol. Lett., vol.11, no.8, pp.1003-1005, 1999.

[4] H. Haeiwa, T. Naganawa, and Y. Kokubun, "Wide range center wavelength trimming of vertically coupled microring resonator filter by direct UV irradiation to SiN ring core," Photonics Technol. Lett., vol.16, no.1, pp.135-137, 2004.

[5] H. Albers, L.T.H. Hilderink, E. Szilágyi, F. Paszti, P.V. Lambeck, and Th.J.A. Popma, "Reduction of hydrogen induced losses in PECVD $\mathrm{SiO}_{x} \mathrm{~N}_{y}$ optical waveguides in the near infrared," Proc. IEEE LEOS’95, pp.88-89, San Francisco, 1995. 
[6] K. Wönhoff, P.V. Lambeck, and A. Driessen, "Design, tolerance, analysis, and fabrication of silicon oxynitride based planar optical waveguides for communication devices," J. Lightwave Technol., vol.17, no.8, pp.1401-1407, 1999.

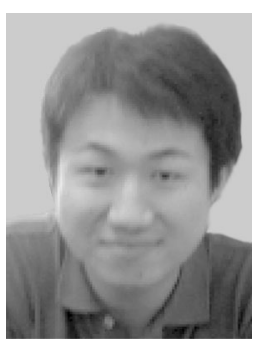

Satoshi Ueno was born in Kanagawa, Japan, on April 7, 1981. He received his B.E. degree in Electrical and Computer Engineering from Yokohama National University, Yokohama, Japan, in 2004. He is working on a microring resonator filter towards an M.E. degree.

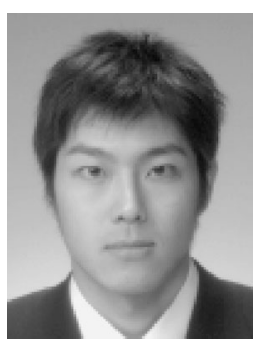

Toshiki Naganawa was born in Aichi, Japan, on November 27, 1979. He received his B.E. degree in Electrical and Computer Engineering from Yokohama National University, Yokohama, Jpapan, in 2003. He is working on a microring resonator filter towards an M.E. degree.

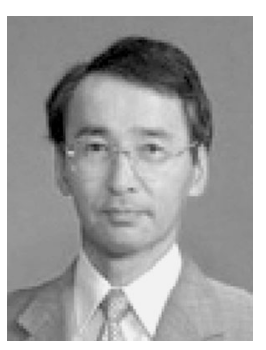

Yasuo Kokubun was born in Fukushima, Japan, on July 7, 1952. He received his B.E. degree from Yokohama National University, Yokohama, Japan, in 1975, and M.E. and PhD Eng. degrees from Tokyo Institute of Technology, Tokyo, Japan, in 1977 and 1980, respectively. After working at the Research Laboratory of Precision Machinery and Electronics, Tokyo Institute of Technology, as a Research Associate from 1980 to 1983 , he joined the Yokohama National University as an Associate Professor in 1983, and is currently a Professor of the Department of Electrical and Computer Engineering. His current research is on integrated photonics, particularly waveguide-type functional devices and three-dimensional integrated photonics. From 1984 to 1985, he was with AT\&T Bell Laboratories, Holmdel, $\mathrm{NJ}$, as a Visiting Reseacher and was engaged on a study of novel waveguide on a semiconducting substrate (ARROW) for integrated optics. From 1996 to 1999 , he served as the project leader of the "three-dimensional microphotonics" project at the Kanagawa Academy of Science and Technology. Professor Kokubun is a member of the Institute of Electrical and Electronics Engineers, the Japan Society of Applied Physics, and the Optical Society of America. 